\title{
Dynamic Thermal Performance Metrics for Adaptive Building Constructions
}

\author{
Ciril Arkar ${ }^{1}$, Marco Perino $^{2}$ \\ ${ }^{1}$ Laboratory for Sustainable Technologies in Buildings, Faculty of Mechanical Engineering, \\ University of Ljubljana, Aškerčeva 6, 1000 Ljubljana, Slovenia \\ ${ }^{2}$ Department of Energy - DENERG, Politecnico di Torino, Corso Duca degli Abruzzi 24, 10129 \\ Torino, Italy
}

\begin{abstract}
Contemporary building envelopes are becoming adaptive and multifunctional in order to further improve the energy efficiency of buildings. However, the selection and characterization of building façade components are still based on traditional metrics - a steady-state $U$-value and g-value, which does not provide information on the improved thermal performance of adaptive building envelopes. Therefore, much effort is invested in the development of new dynamic performance metrics. This paper presents the development of a new dynamic thermal performance metric $U$, which is determined from numerical results of the building construction thermal response (the inner surface heat flux and the air temperature difference data) at the Sol-air outdoor boundary conditions. The results of the analysis have shown that by adaptive building construction the new metric $U^{\prime}$ is lower than the construction $U$-value; it decreases with the decreased daily heat transfer and with the increased specific heat capacity. Further research and development of the proposed $U^{\prime}$ metric is needed for adaptive building constructions with low $U$-value and at boundary conditions, which result in the daily heat gains.
\end{abstract}

\section{Introduction}

A building envelope represents the dividing line between the indoor and outdoor environment. In a traditional form, this is a static element, which, if properly thermally insulated, strongly hinders the utilization of the potential of the outdoor and indoor environment, and thus the possibility of further reduction of the energy use in buildings. It is therefore recognized by the professionals as the key element for achieving the energy and climate goals set in the EU. In recent years, a lot of effort has been invested in the research and development of the multifunctional and adaptive building envelopes, which enables greater exploitation of energy potential of the outdoor environment and thus significantly improves buildings' energy efficiency, while at the same time provides improved living comfort in buildings and in the urban environment. However, one of the greatest challenges is the selection of metrics, which would, in a simple, understandable and useful way, present their thermal response and energy performance.

Thermal performance of traditional - static and opaque building envelope element is characterized with thermal transmittance or $U$-value; a basic indicator, which is calculated at steady-state conditions. This metric is the basic parameter (for opaque building constructions), used in engineering tools for buildings' energy performance calculations, using monthly (and even hourly) calculation methods (Verbeke and Audenaert, 2018). It is also often the only building construction parameter in regression models for prediction of energy use in buildings (Lam et al., 2010). It is therefore not surprizing that $U$-value is a basic performance metric, used when comparing or selecting different constructions. It is also a performance metric, which is easily recognized by building designers, stakeholders and experts (Arkar et al., 2018; Favino et al., 2016). As demonstrated by several researches (Alterman et al., 2012; de Gracia et al., 2011), static $U$-value does not present a good indicator for actual energy need for building heating or cooling, as it does not encompass the dynamic thermal properties of building's construction. Favino et al. (2016) also established that $U$-value is not as useful in presenting the benefit of adaptive and multifunctional building constructions.

To characterize the thermal performance of multifunctional and adaptive building envelopes, researchers proposed several tailored, non-standardized performance metrics. To further emphasize the enhanced thermal performance of adaptive building envelope construction, 'effective' (also dynamic or equivalent) thermal transmittance is often determined using realistic or idealised dynamic boundary conditions, including solar irradiance on the building construction (Arkar et al., 2018a; Bellamy, 2014; Bianco et al., 2017; Favino et al., 2016; Reilly and Kinnane, 2017). Less indicative, but presenting the same characteristic, are for example reduction of heat losses (Arkar et al., 2018b) or dynamic benefit (Moody and Sailor, 2013). Alterman et al. (2012) proposed a dynamic thermal performance metric called $\mathrm{T}$ value, which is tailored for free-floating indoor conditions. T-value is determined from diurnal outer and inner surface temperature plot, which has a typical ellipse shape. T-value is defined as an angle of ellipse principle axes. As demonstrated, the T-value reflects construction's insulation and thermal mass properties. The literature review further reveals that periodic thermal transmittance and decrement factor, determined according to standard EN ISO 13786 or using extended admittance method, are most often used to evaluate building construction's dynamic thermal properties at periodic outdoor boundary conditions (Černe and Medved, 2005; de Gracia et al., 2011; Gasparella et al., 2011; Thiele et al., 2017). 
However, this analytical method is only appropriate for building constructions composed of materials with constant thermo-physical properties. Although there were some attempts to use thermal admittance method for adaptive building constructions (Thiele et al., 2017), it could be concluded that further development of dynamic performance metrics is required for building constructions, incorporating phase change material (PCM) and other adaptive and multifunctional envelopes.

This study presents the attempt of development of a new and relatively simple dynamic thermal performance metric, suitable for adaptive, as well as standard, building envelopes. The metric was developed based on the critical evaluation of the T-value metric and considering the basis of thermal admittance method. To evaluate dynamic thermal performance of adaptive envelopes, numerical simulations, using validated finite element numerical model, was used. New metric, which was developed and evaluated, was marked as $U^{\prime}$ and proposed for further consideration and development. The development of new metric and a case study analyses are presented for a building construction with a PCM layer.

\section{Methods}

\section{Dynamic thermal performance metric development}

For the assessment of building construction dynamic thermal characteristics, metrics, specified within standard EN ISO 13786:2017, prevails. One of the most often used in analyses is periodic thermal transmittance $\left(Y_{n m}\right)$, here within marked as $U_{p}$, which is defined as a ratio of complex inner surface heat flux $\hat{q}_{i}$ and complex amplitude of ambient air temperature; considering constant indoor air temperature and sinusoidal outdoor air temperature variation. Several simple software tools exist, made according to analytical thermal admittance method, which enable building designers relatively fast and simple analyses of building constructions dynamic properties. Building construction's periodic thermal transmittance $U_{p}$ can be also determined from numerical thermal response results as:

$$
U_{p}=\left(\dot{q}_{i, \max }-\dot{q}_{i, \min }\right) /\left(T_{e, \max }-T_{e, \min }\right)
$$

where $\dot{q}_{i}$ is the inner surface heat flux and $T_{e}$ is the ambient air temperature. The same result is obtained as if calculated with complex matrixes. Obtained results are the same for any amplitude of ambient air temperature $\left(A_{e}\right)$ and any average ambient air temperature $\left(T_{e, a v}\right)$. Practically, the same $U_{p}$ is also obtained if 'Sol-air' temperature is used as the outdoor boundary condition. This means that $U_{p}$ could not give any indication on energy, required to maintain specified indoor air temperature conditions. As it is evident from equation (1) and demonstrated later in Figure 1 , the $U_{p}$ actually indicates to inner surface heat flux variation. It should also be noted that $U_{p}$ does not allow indoor air temperature variation. In case of temperature dependent material properties or other adaptive properties, $U_{p}$ can be precisely determined only from numerical results of the construction thermal response.
Another metric, the dynamic temperature response - Tvalue, introduced by Alterman et al. (2012), takes into account diurnal thermal response of building construction. So it does not encompass just daily max and min value, as in case of $U_{p}$ (equation (1)). T-value is derived from diurnal outer and inner surface temperature variation. The T-value metric has the unit of temperature, which is less indicative. It also requires normalisation, which additionally complicates the evaluation of building constructions.

New dynamic performance metric $U$ is determined from the diurnal inner surface heat flux $\left(\dot{q}_{i}\right)$ and indoor and outdoor air temperature difference $\left(T_{e}-T_{i}\right)$ data. As demonstrated in Figure 1, in the case of sinusoidal outdoor boundary conditions, the inner surface heat flux forms an elliptical shape on the $\dot{q}_{i} / d T$ chart. The shape depends on the construction's specific heat capacity $C$. In the special case of a "no mass" construction $\left(C=0 \mathrm{~kJ} / \mathrm{m}^{2} \mathrm{~K}\right)$, the ellipse flattens to a line (red dots in Figure 1). Linear fit to these data $(y=a+b \cdot x)$, considering air temperature difference $T_{e}-T_{i}$ as independent variable and inner surface heat flux $\dot{q}_{i}$ as dependent variable, gives intercept value of 0 and the slope which equals the construction's $U$-value.

Linear regression is one of the simplest statistics that presents relation between two variables. Linear regression can handle any boundary conditions and any shape of obtained $\dot{q}_{i}-d T$ numerical data, which are not elliptical in case of adaptive building construction or at real (measured) boundary conditions. Therefore, for real building constructions, linear fit to numerical data was used $(y=b \cdot x)$, considering forced intercept trough the origin $(a=0)$. Slope of the line with zero intercept was selected as the new building construction dynamic performance metric. It has the unit of thermal transmittance and is marked in this paper as $U^{\prime}$.

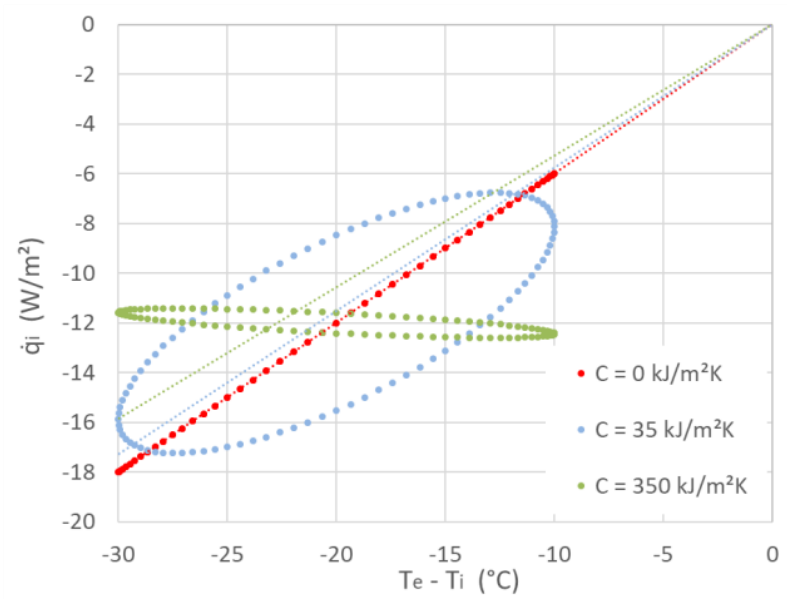

Figure 1: Diurnal inner surface heat flux variation of a building construction with $U=0.6 \mathrm{~W} / \mathrm{m}^{2} \mathrm{~K}$ and different specific heat capacity $C$; boundary conditions: $T_{i}=20$ ${ }^{\circ} \mathrm{C}, A_{i}=0 \mathrm{~K}, T_{e, a v}=0{ }^{\circ} \mathrm{C}, A_{e}=10 \mathrm{~K}$.

As it is evident from Figure 1 and Table 1 , the $U$ value decreases with increasing building construction specific heat capacity $C$. It is close to the construction $U$-value $(0.6$ $\mathrm{W} / \mathrm{m}^{2} \mathrm{~K}$ ) in case of lightweight building construction and 
reduces for slightly more than $10 \%$ in case of heavyweight building construction. The $U_{p}$ reduces much more (Table 1) and indicates more to thermal response dynamics and not to the energy related characteristics of building construction.

Table 1: Ú and $U_{p}$ values for case-study construction presented in Figure 1.

\begin{tabular}{|l|c|c|}
\hline & $\dot{U}\left(\mathbf{W} / \mathbf{m}^{2} \mathbf{K}\right)$ & $\boldsymbol{U}_{\boldsymbol{p}}\left(\mathbf{W} / \mathbf{m}^{2} \mathbf{K}\right)$ \\
\hline$C=0 \mathrm{~kJ} / \mathrm{m}^{2} \mathrm{~K}$ & 0.60 & 0.60 \\
\hline$C=35 \mathrm{~kJ} / \mathrm{m}^{2} \mathrm{~K}$ & 0.576 & 0.524 \\
\hline$C=350 \mathrm{~kJ} / \mathrm{m}^{2} \mathrm{~K}$ & 0.529 & 0.059 \\
\hline
\end{tabular}

\section{Building construction and boundary conditions}

To further evaluate new building constructions' dynamic performance metric $U^{\prime}$, a case study building construction was selected. The building construction has a $U$-value of $0.4 \mathrm{~W} / \mathrm{m}^{2} \mathrm{~K}$, which represents a moderately thermally insulated outer wall. Thermal insulation layer is placed on the outer side of the wall. Specific heat $c_{p}$ of wall material varies in analysis according to set building construction overall specific heat capacity $C$. This is a case study construction with constant thermo-physical properties. A case study adaptive building construction has the same $U$ value and additional $2 \mathrm{~cm}$ thick PCM layer on inner side of the construction. A PCM with a narrow $2 \mathrm{~K}$ melting temperature range and a latent heat of fusion of $100 \mathrm{~kJ} / \mathrm{kg}$ is assumed for analyses. In the numerical model, phase change process is modelled considering apparent specific heat for the PCM. The apparent specific heat of the PCM was approximated using two polynomial equations and anticipating no hysteresis.

In the analysis, dynamic boundary conditions were considered, as presented in Figure 2. On the outer side, a Sol-air temperature boundary conditions were used, considering different average daily ambient air temperature $T_{e, a v}$, amplitude of ambient air temperature variation of $5 \mathrm{~K}\left(A_{e}=5 \mathrm{~K}\right)$ and an incident solar radiation profile with a daily solar irradiation $H$ of $2.5 \mathrm{kWh} / \mathrm{m}^{2}$ day.

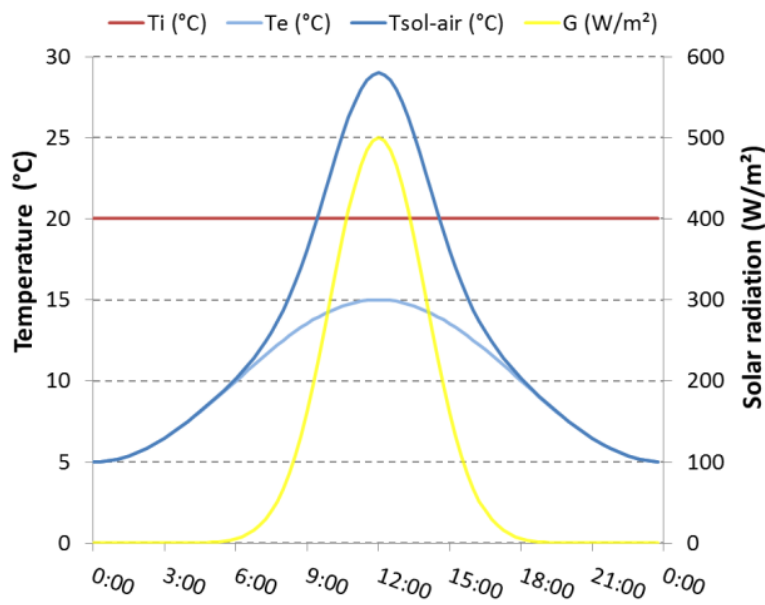

Figure 2: Indoor and outdoor temperature and solar radiation boundary conditions adopted for analysis; a case with $T_{e, a v}=10^{\circ} \mathrm{C}$; parameters that do not change: $T_{i}=20^{\circ} \mathrm{C}, A_{i}=0 \mathrm{~K}, A_{e}=5 \mathrm{~K}, \mathrm{H}=2.5 \mathrm{kWh} / \mathrm{m}^{2}$ day.

$$
T_{\text {sol-air }}=T_{e}+\alpha_{s} \cdot G / h_{e}
$$

$T_{\text {sol-air }}$ (2) was calculated considering solar absorption $\alpha_{s}$ of 0.7 and outer surface heat transfer coefficient $h_{e}$ of 25 $\mathrm{W} / \mathrm{m}^{2} \mathrm{~K}$. Constant indoor air temperature of $20{ }^{\circ} \mathrm{C}$ was considered in all simulations, as shown in Figure 2.

\section{Results}

A validated transient one-dimensional heat transfer numerical model (Arkar et al., 2018a) with a time step of 15 min was used for numerical analysis. Three days with the same boundary conditions were used in simulation; the first two days were required to obtain appropriate initial conditions, while the simulation results of the third day was used for dynamic metrics calculation. Beside $U_{p}$ and $U$ metrics, daily heat losses $q_{i}$ (3), calculated from the daily inner surface heat flux values, was also determined:

$$
q_{i}=\Sigma\left(\dot{q}_{i} \cdot d t\right)
$$

In the first analysis, thermal response of homogeneous wall (without PCM layer) with $C=100 \mathrm{~kJ} / \mathrm{m}^{2} \mathrm{~K}$ at different outdoor air boundary conditions $\left(T_{e, a v}\right.$ of $10^{\circ} \mathrm{C}$, $5{ }^{\circ} \mathrm{C}$ and $0{ }^{\circ} \mathrm{C}$ ) was determined. Results are presented in Figure 3. It can be seen that due to non-sinusoidal outdoor $T_{\text {sol-air }}$ boundary conditions, the shape of inner surface heat flux is not elliptical as in Figure 1. Higher (negative) heat flux toward the ambient is observed at lower average ambient air temperatures (higher temperature differences). Linear regression trough the origin gives the $\dot{U}$ values of $0.199 \mathrm{~W} / \mathrm{m}^{2} \mathrm{~K}, 0.294 \mathrm{~W} / \mathrm{m}^{2} \mathrm{~K}$ and 0.339 $\mathrm{W} / \mathrm{m}^{2} \mathrm{~K}$ at temperature differences $T_{i}-T_{e, a v}$ of $10 \mathrm{~K}, 15 \mathrm{~K}$ and $20 \mathrm{~K}$ respectively. The periodic thermal transmittance $U_{p}$ value is $0.186 \mathrm{~W} / \mathrm{m}^{2} \mathrm{~K}$ at all three different boundary conditions. From Figure 3 and presented results it could also be concluded that with increasing temperature differences $T_{i}-T_{e, a v}$, the daily heat losses, as well as the $U^{\prime}$ value, increase.

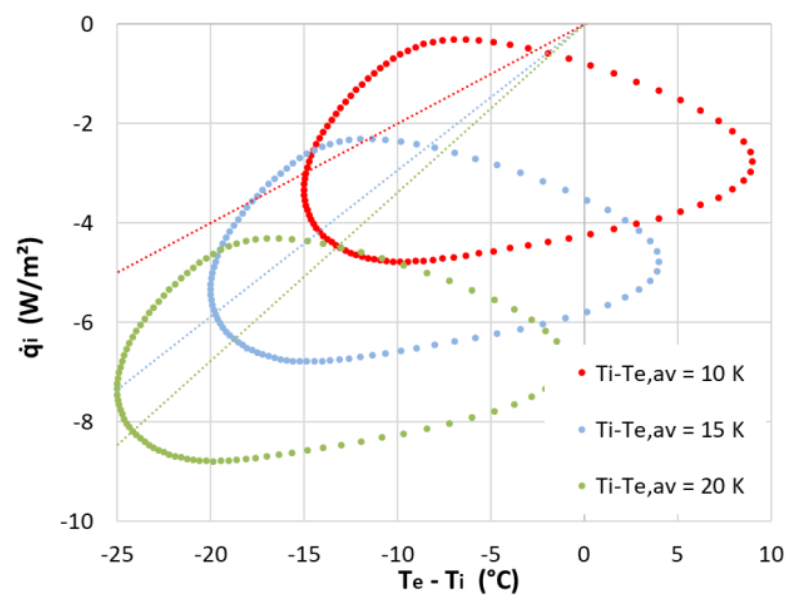

Figure 3: Daily variation of inner surface heat flux $\dot{q}_{i}$ and linear regression trough the origin lines for a homogeneous wall at three different $T_{\text {sol-air }}$ boundary conditions (2); $U=0.4 \mathrm{~W} / \mathrm{m}^{2} \mathrm{~K}$, constant thermophysical properties of wall layers.

The $U$ values, as well as $U_{p}$ values, were determined for homogeneous building construction of different specific heat capacities and for different outdoor $T_{\text {sol-air }}$ boundary conditions by varying the average ambient air temperature 
$T_{e, a v}$. Results are presented in Figure 4. The results showed that the $\dot{U}$ value depends on the building construction's $C$ value and on boundary conditions. Meanwhile, the $U_{p}$ value is not affected by boundary conditions. Correlation between the $U$ values and the construction daily heat losses $q_{i}$ is presented in Figure 5. It can be seen that it would be possible to derive some regression model, however, the model would need to include information on building construction specific heat capacity $C$. From the presented results, it can be concluded that for homogeneous building constructions, composed of layers with constant thermo-physical properties, the $U^{\prime}$ metric provides more relevant information on the building construction thermal performance than the $U_{p}$ metric.

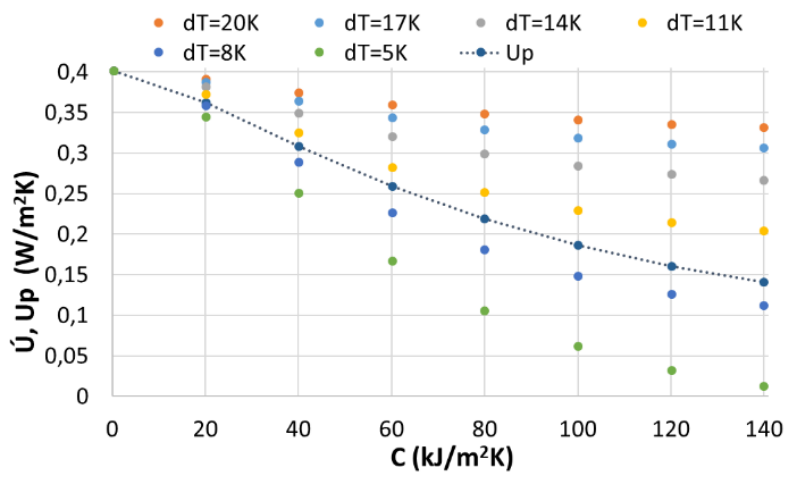

Figure 4: New dynamic thermal performance metric $U$ and periodic thermal transmittance $U_{p}$ values at different outdoor $T_{\text {sol-air }}$ boundary conditions; $d T=T_{i}$ $T_{e, a v}$; wall with $U=0.4 \mathrm{~W} / \mathrm{m}^{2} \mathrm{~K}$.

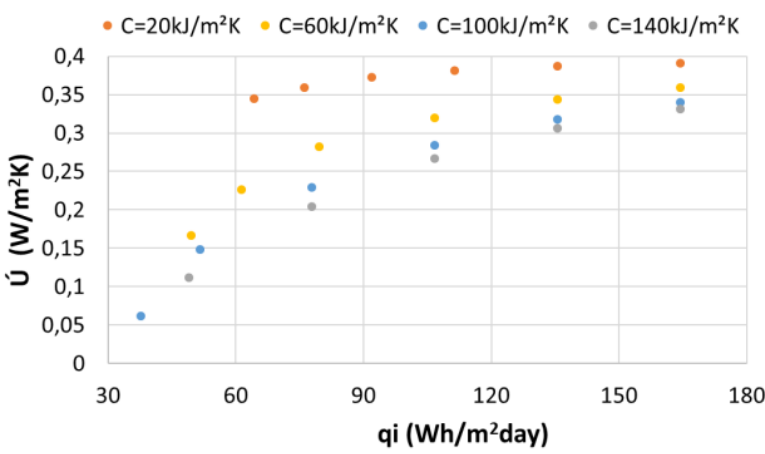

Figure 5: Correlation between $U$ and daily heat losses $q_{i}$ determined at different boundary conditions; wall with $U$ $=0.4 \mathrm{~W} / \mathrm{m}^{2} \mathrm{~K}$.

As the primary goal is to develop a dynamic thermal performance metric for adaptive and multifunctional building constructions, an analysis was also made for such construction. A construction with a PCM layer, which ensures an adaptive thermal response, was used for the analysis. Thermal response of such adaptive building construction, considering three different PCM peak melting temperatures, is presented in Figure 6. Red dots are presenting the inner surface heat flux in case when there is no phase change in the PCM layer, due to improper PCM phase change temperature range. In case of PCM with $T_{m}=20.5^{\circ} \mathrm{C}$, only a part of the latent heat can be exploited, as can be concluded from calculated $\dot{q}_{i}$ values (Figure 6). The third PCM, with optimal peak melting temperature of $19{ }^{\circ} \mathrm{C}$, enables latent heat accumulation and in this way considerably increases specific heat capacity of adaptive building construction, which can be seen from the calculated thermal response. Linear regression lines (Figure 6) or determined $U$ values (Table 2) indicate on improved dynamic thermal response due to the phase change process within the building construction.

Table 2: Ú and Up values for adaptive building construction (case-study presented in Figure 6).

\begin{tabular}{|c|c|c|}
\hline & $\dot{U}\left(\mathbf{W} / \mathbf{m}^{2} \mathbf{K}\right)$ & $\boldsymbol{U}_{\boldsymbol{p}}\left(\mathbf{W} / \mathbf{m}^{2} \mathbf{K}\right)$ \\
\hline$T_{m}>T_{e}$ & 0.341 & 0.297 \\
\hline$T_{m}=20.5^{\circ} \mathrm{C}$ & 0.326 & 0.254 \\
\hline$T_{m}=19^{\circ} \mathrm{C}$ & 0.273 & 0.025 \\
\hline
\end{tabular}

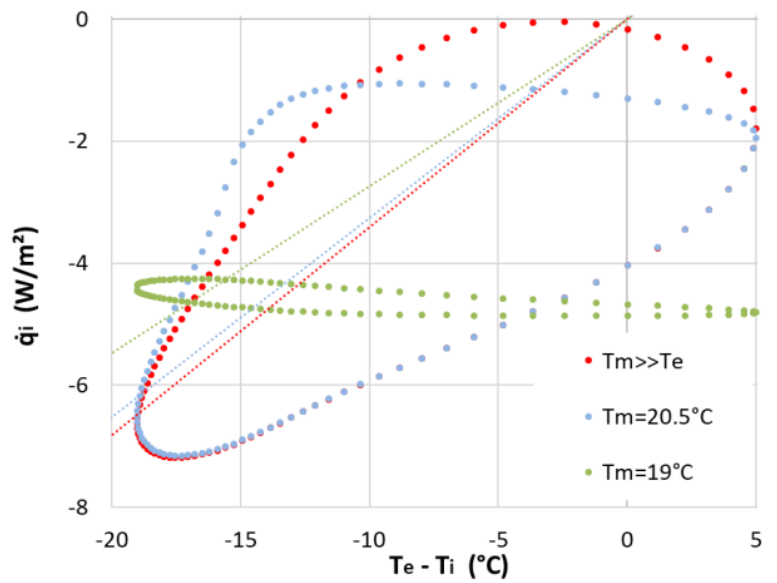

Figure 6: Daily variation of inner surface heat flux $\dot{q}_{i}$ and linear regression trough the origin lines for an adaptive building construction with a PCM layer with different peak melting temperature $T_{m} ; U=0.4 \mathrm{~W} / \mathrm{m}^{2} \mathrm{~K}$;

$$
T_{e, a v}=6{ }^{\circ} \mathrm{C} \text {. }
$$

Further analysis was performed for different outdoor $T_{\text {sol-air }}$ boundary conditions (different $T_{e, a v}$ ) and considering different peak melting temperatures of the PCM layer of adaptive building construction. Lightweight construction with specific heat capacity of $50 \mathrm{~kJ} / \mathrm{m}^{2} \mathrm{~K}$ was selected for the analysis. Calculated $U$ and $U_{p}$ values are presented in Figure 7. Latent heat accumulation or phase change process can easily be noticed from $U_{p}$ results, as it was found that $U_{p}$ value does not depend on boundary conditions. Nevertheless, improved thermal response can also be recognized from presented $U$ results, which also depends on used boundary conditions, if only results at selected boundary conditions are compared. The results indicate that improved dynamic thermal response is obtained if PCM with peak melting temperature of $19{ }^{\circ} \mathrm{C}$ or $20{ }^{\circ} \mathrm{C}$ is used. At some boundary conditions, a PCM with $T_{m}=21^{\circ} \mathrm{C}$, also performs well. 

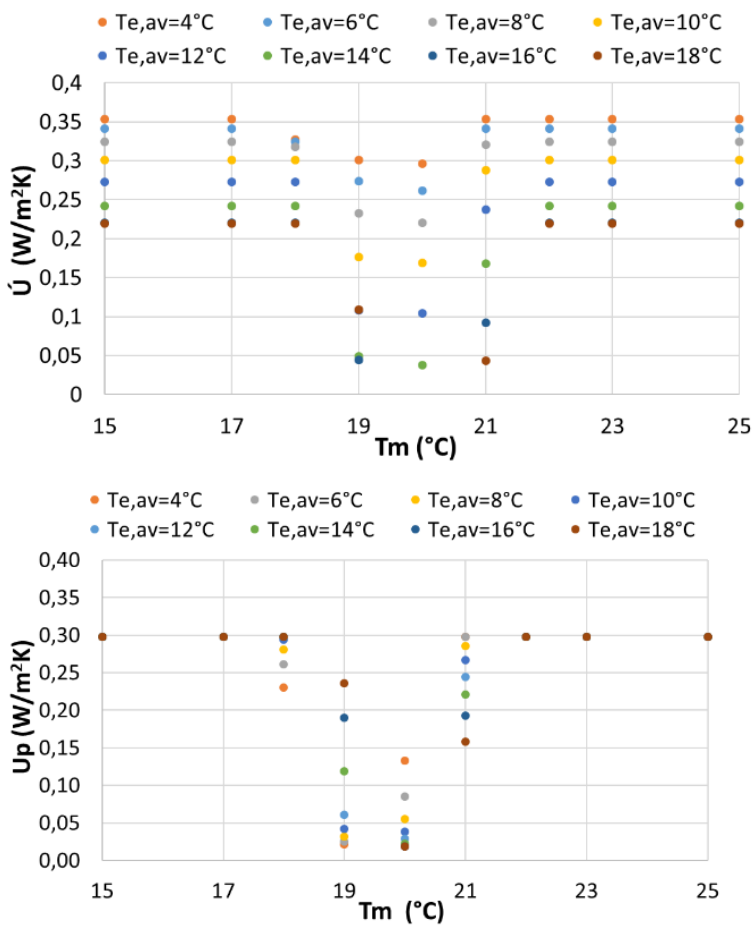

Figure 7: $U$ and $U_{p}$ values of adaptive building construction with PCM layer with different peak melting temperatures $T_{m}$ at different outdoor $T_{\text {sol-air }}$ boundary conditions; $U=0.4 \mathrm{~W} / \mathrm{m}^{2} \mathrm{~K}, C=50 \mathrm{~kJ} / \mathrm{m}^{2} \mathrm{~K}$.

The dynamic performance metric values and construction daily heat losses were compared next. The results are presented in Figure 8.
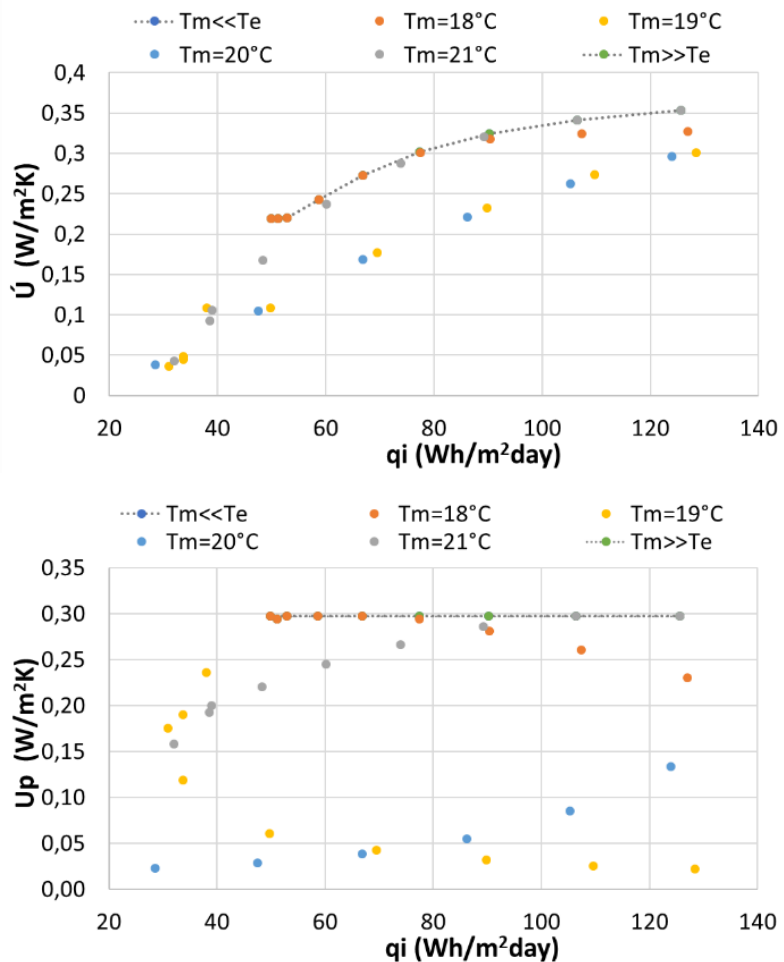

Figure 8: Correlation between $U$ or $U_{p}$ and daily heat losses $q_{i}$ determined at different boundary conditions; $U$ $=0.4 \mathrm{~W} / \mathrm{m}^{2} \mathrm{~K}, \mathrm{C}=50 \mathrm{~kJ} / \mathrm{m}^{2} \mathrm{~K}$.
The dots with a dashed line present the case of static building construction (no phase change in PCM layer). The dots without the line present adaptive building construction with PCM with suitable $T_{m}$. From the presented $U^{\prime}-q_{i}$ results it could be estimated which PCM undergoes complete phase change process and for which PCMs boundary conditions enable only partial utilization of the latent heat of fusion. In analogy with Figure 5, the effective $C$ could be determined for adaptive building construction. It would also be possible to compare static and adaptive construction or evaluate 'adaptiveness' of construction thermal response. From the $U_{p}-q_{i}$ chart (Figure 8 bottom) no such comparison or correlation can be derived.

\section{Conclusion}

Thermal performance of a building depends on dynamics of internal and solar heat gains, as well as on the dynamic thermal response of building constructions. In the case of adaptive and multifunctional building constructions, the thermal response can be much different than by traditional building constructions. Nevertheless, current building design approach is still governed by construction's $U$ value, which is a static building construction's parameter, widely recognized by building designers, stakeholders and experts. The starting point of this research was therefore the development of a new dynamic performance metric, which would be easily recognized among professional public and would include information on building construction's dynamic thermal response and energy performance.

Development of proposed $U$ metric was based on the Tvalue indicator, which considers daily thermal response of building construction at real boundary conditions. However, instead of a surface temperature, the inner surface heat flux was selected as parameter, which is also used for $U$-value and periodic thermal transmittance calculation. As adaptive building envelopes enable greater solar energy utilization, the sol-air temperature was selected as the outdoor boundary condition. The proposed $U$ dynamic thermal performance metric has the unit of the $U$-value. Its value is obtained from the linear fit through the origin to daily thermal response data.

Performed analysis demonstrated that developed $U$ metric is associated with the building construction $U$-value. It equals the construction $U$-value in case of "no mass" construction. It decreases with the increased building construction specific heat capacity. By adaptive building constructions, it further decreases with the enhanced thermal response. It depends on boundary conditions and decreases with decreased daily heat transfer. As demonstrated in the analysis, the $U$ metric can indicate on static or adaptive performance of building construction.

In further development of the proposed dynamic performance metric $U$, there is a need to define the exact boundary conditions and explore the possibility of using real boundary conditions and measured thermal response of adaptive building envelopes. This would allow the evaluation of processes of adaptive building constructions, that are more difficult to model with the 
numerical models (PCM hysteresis and subcooling, plants evapotranspiration of green envelopes, etc.). The metric needs further development also in terms of testing of statistical significance of obtained results, as well as on results interpretation and correlation to static $U$-value and construction or building energy performance.

\section{Acknowledgement}

The authors would like to acknowledge the financial support provided by the Slovenian Research Agency within the research program P2-0223 (C) "Heat and Mass Transfer" and the networking opportunities given by the Cost Action TU1403 "Adaptive Façades Network".

\section{References}

Alterman D. Moffiet T. Hands S. Page A. Luo C. and Moghtaderi B. (2012) A concept for a potential metric to characterise the dynamic thermal performance of walls. Energy and Buildings 54, 52-60.

Arkar C. Domjan S. and Medved S. (2018a) Lightweight composite timber façade wall with improved thermal response. Sustainable Cities and Society 38, 325-332.

Arkar C. Domjan S. and Medved S. (2018b) Heat transfer in a lightweight extensive green roof under waterfreezing conditions. Energy and Buildings 167, 187199.

Belamy L. (2014) Towards the development of new energy performance indicators for the external walls of residential buildings. Energy and Buildings 68, 696-702.

Bianco L. Cascone Y. Goia F. Perino M. Serra V. (2017) Responsive glazing systems: Characterization methods and winter performance. Solar Energy 155, 372-387.

Černe B. and Medved S. (2005) The dynamic thermal characteristics of lightweight building elements with a forced ventilated cavity and radiation barriers. Energy and Buildings 37, 972-981. de Gracia A. Castell A. Medrano M. and Cabeza L.F. (2011) Dynamic thermal performance of alveolar brick construction system. Energy Conversion and Management 52, 2495-2500.

Favoino F. Goia F. Perino M. and Serra V. (2016) Experimental analysis of the energy performance of an ACTive, RESponsive and Solar (ACTRESS) façade module. Solar Energy 133, 226-248.

Gasparella A. Pernigotto G. Baratieri M. Baggio P. (2011) Thermal dynamic transfer properties of the opaque envelope: Analytical and numerical tools for the assessment of the response to summer outdoor conditions. Energy and Buildings 43, 2509-2517.

International Organisation for Standardisation (2017). Thermal performance of building components Dynamic thermal characteristics - Calculation methods (EN ISO 13786:2017).

Lam J.C. Wan K.K.W. Liu D. and Tsang C.L. (2010) Multiple regression models for energy use in airconditioned office buildings in different climates. Energy Conversion and Management 51, 2692-2697.

Moody S.S. and Sailor D.J. (2013) Development and application of a building energy performance metric for green roof systems. Energy and Buildings 60, 262269

Reilly A. and Kinnane O. (2017) The impact of thermal mass on building energy consumption. Applied Energy 198, 108-121.

Thiele A. M. Liggett R. S. Sant G. and Pilon L. (2017) Simple thermal evaluation of building envelopes containing phase change materials using a modified admittance method. Energy and Buildings 145, 238 250.

Verbeke S. and Audenaert A. (2018) Thermal inertia in buildings: A review of impacts across climate and building use. Renewable and Sustainable Energy Reviews 82, 2300-2318. 